\title{
EL HOMBRE COMO "SECUNDUS DEUS": FORMA ÚNICA Y RECONSTRUCCIÓN NOCIONAL DE GÉNEROS Y ESPECIES EN EL PENSAMIENTO CUSANO
}

Claudia D'Amico*

SÍNTESIS - La metafísica de Nicolás de Cusa presentada, por primeira vez, en su obra capital De docta ignorantia (1440) presenta como una de sus nociones fundantes la idea de Máximo absoluto según la cual la maximidad, por ser tal, carece de toda relación. Así pues, el Máximo absoluto debe ser concebido en coincidencia con su opuesto, el mínimo absoluto - coincidentia oppositorum - y, al mismo tiempo, puesto que nada se le opone, co-implicando en sí mismo todas las cosas - complicatio absoluta - sin ser ninguna de ellas de modo singular. De esta manera, el Máximo resulta la forma única universal de todo lo real y lo posible. las ideas en el Lógos divino, noción que atraviesa diversas expresiones del neoplatonismo cristiano, aparecen aquí unificadas e igualadas en una única esencia, que es el mismo Logos, que "corre" por todas las cosas y es su quididad absoluta. Esta presentación nos conduce necesariamente a la pregunta por la constitución de la singularidad y por la predicación universal acerca de estoa singulares. A la primera pregunta responde Nicolás con la doctrina de la "contracción"; a la segunda debemos responder nosotros reconstruyendo la idea cusana de des-velamiento del muno que lleva a cabo la humana mens a partir del entramado nocional de géneros y especies. Esta construcción nocional llevará al Cusano a llamar al hombre "segundo dios".

PALABRAS-CLAVE - Nicolas de Cusa. Hombre. Diós. Forma. Género y especie.
ABSTRACT - The metaphysics of Nicholas of Cusa, presented for the first time in their capital work "De docta ignorantia" (1440) presents like one of their fundamental notions the idea of absolute maximum according to which the Maximality, to be such, lacks of all relationship. Therefore, the absolute maximum should be conceived in coincidence with its opposed one, the minimum absolute - "coincidentia oppositorum" - and, at the same time, since anything is opposed to it, co-implying in itself all the things "coimplicati absoluta" without being none of them in a singular way. This way, the Maximum is the universal unique form of all the real things and the possible things. The ideas in the divine logos, notion that crosses diverse expressions of Christian Neoplatonism, appears here unified and equaled in an only essence that is the same Logos that "runs" for all the things and it is its absolute quiddity. This presentation necessarily leads us to the question for the constitution of the singularity and for the universal preaching about these singular ones. To the first question Nicholas responds with the doctrine of the "contraction"; to the second we should respond reconstructing the cusan idea of un-veiling of the world that carries out the human "mens" starting from the notional lattice of genre and species. This notional consruction it will take the Cusan to call to the man "second god".

KEY WORDS - Nicolas of Cusa. Man. God. Form. Gernus and species.

* Universidad de Buenos Aires.

\begin{tabular}{|l|l|l|l|l|l|}
\hline VERITAS & Porto Alegre & v. 44 & n. 3 & Setembro 1999 & p. 815-822 \\
\hline
\end{tabular}


Lo máximo absoluto concebido como aquello mayor que lo cual nada puede haber atraviesa distintas manifestaciones del pensamiento medieval. Sin embargo, esta fórmula se presenta en el pensamiento de Nicolás de Cusa con una radicalidad tal que las consecuencias que de su interpretación habrán de derivarse darán cuenta no sólo de la relación entre la unidad y la multiplicidad y de la conformación ontológica de lo real, sino también de la posibilidad de su conocimiento y la de su predicabilidad. Las relaciones entre Dios y el mundo, la verdad y el hombre, las palabras y las cosas dependen de la definición cusana de maximidad.

En efecto, Nicolás establece que el máximo absoluto es tal porque carece de toda relación. Precisamente, el hecho de ser absoluto significa des-vinculado. ${ }^{1} \mathrm{De}$ este modo, nada puede oponérsele. Así, el máximo absoluto coincide necesariamente con su opuesto, el mínimo absoluto. He aquí la gran intuición del pensamiento cusano: pensar la maximidad es entender de un solo golpe la coincidentia oppositorum. La coincidencia de opuestos en el máximo, entonces, no sólo pone en evidencia su absoluta unidad y simplicidad sino también la sobreabundancia de su naturaleza: afirmar que en lo máximo los opuestos son coincidentes, conduce necesariamente a sostener que nada es fuera de él, puesto que cualquier cosa que puede darse se ha de dar entre estos dos extremos absolutos. De esta manera, esta unidad simple y absoluta es en acto todo posible ser. La posibilidad pura de ser de todas las cosas en el máximo las vuelve a ellas mismas absolutas en él, esto es carente de toda relación o determinación ${ }^{2}$. Esta infinita unidad en cuanto que reúne o co-implica de manera absoluta, desvinculada, todas las cosas es llamado por Nicolás de Cusa complicatio absoluta, Vemos, pues, hasta qué punto las denominaciones de "coincidencia de opuestos" y "complicación absoluta" son complementarias y dependen de la radicalización de la idea de maximidad. ${ }^{3}$

Con todo, es necesario hacer notar que cuando se hace referencia a la unidad infinita en términos de "complicatio", se señala a la misma en tanto principiocausa de lo finito que resulta, por su parte, su explicitación o explicatio. No podría ser de otro modo si, como hemos dicho, en este absoluto se encuentra en acto todo posible ser: asi, a un Dios que contiene todas las cosas co-implicadas en su unidad, le corresponde un mundo que resulta su propio despliegue o "explicación".

La terminología elegida por Nicolás de Cusa para referirse al lazo que une lo causado con su principio-causa excede los límites de la mera causalidad eficiente.

\footnotetext{
"absolutus-a-um" es utilizado frecuentemente por Nicolás como adjetivo, participio del verbo "absolvere" (desatar, desligar...) Esta significación refuerza el sentido de "absoluto-desvinculado" del Dios cusano.

2 El capítulo segundo del primer libro del De docta ignorantia presenta en una síntesis magistral las nociones a las que hacemos referencia, DI, I, 2, 5: "Maximum autem hoc dico, quo nihil maius esse potest. Abundantia vero uni convenit. Coincidit itaque maximitati unitas, quae est et entitas, quod, si opsa talis unitas ab omni respectu et contractione universaliter est absoluta, nihil sibi opponi manifestum est, cum sit maximitas absoluta. Maximum itaque absolutum unum est quod est omnia; in quo omnia, quia maximum. Et quoniam nihil sibi opponitur, secum simul coincidit minimum. Quaere et in omnibus. Et quia absolutum, tunc est actu omne possibile esse, nihil a rebus contrahens, a quo omnia".

3 DI, II, 3, 105: "Maximum autem est cui nihil potest opponi: ubi et minimum est maximum. Unitas igitur infinita est omnium complicatio".
} 
La causalidad de la que se trata es la propia de una causa formal que es, al mismo tiempo, principio es decir que se prolonga como presencia en lo principiado. Más aún, la esencia de la multiplicidad es esta infinita unidad explicitada en ella a tal punto que si la elimináramos los entes parecerían convertirse en una pura nada. ${ }^{4}$ La infinita unidad es pues la absoluta causa formal que resulta la esencia misma de todas las cosas. Esta forma ejemplar que las constituye a todas, puesto que es infinita, no puede ser múltiple sino una. En efecto, afirma el Cusano que si el infinito tuviera partes cada una de ellas deberia ser infinita por lo cual todo en el infinito es uno y, además, igual. ${ }^{5}$ Así, el mundo en cuanto explicatio dei tiene una forma única, común a todas las cosas y que puede por esto mismo ser denominada "igualdad" en el sentido en que no está sujeta al más y al menos pues en ella estos opuestos coinciden. ${ }^{6}$

La postulación de un único ejemplar o complicación absoluta para la diversidad de las cosas en razón de su carácter de infinita coincidencia de opuestos, no es para el Cusano mismo - aunque sí para sus intérpretes - una originalidad de su sistema. Para él, el propio Platón habría dicho que el ejemplar era uno considerado en sí aunque parezcan diversos ejemplares cuando los consideramos con relación a las cosas." Ahora bien, una lectura apresurada de las afirmaciones cusanas acerca de que el máximo es todas las cosas y que ese máximo infinito se explicita en lo finito, podria conducirnos a interpretar erróneamente el pensamiento cusano tal como lo hizo Juan Wenck en su propio tiempo quien acusó a Nicolás de panteismo. Ante esto el propio cusano responde afirmando que el modo en que dichas cosas son contenidas es idéntico a la manera en que lo causado está en su causa. ${ }^{8}$ Complicadas en una causa que es coincidencia de opuestos, las cosas son allí sin alteridad, una y la misma con su causa, de alli la unicidad de la forma o necessitas rerum. Esto, sin embargo, no da cuenta de la manera en que la totalidad de lo real, constituído por una única forma essendi que no es otra que el máximo absoluto explicitado como mundo, se determina y constituye en los variados modos de la multiplicidad. En otras palabras: la complicación absoluta de todas las cosas en su principio y, a la vez, la presencia de este principio como la unidad oculta de la multiplicidad no permite justificar esta multiplicidad en cuanto tal: los entes no reciben su pluralidad de la unidad.

Resulta imprescindible, pues, para completar el cuadro de la relación entre lo finito y lo infinito, poner en juego junto a las nociones de complicatio y explicatio, otra típicamente cusana: la noción de "contracción"(contractio).

\footnotetext{
4 DI, I, 17, 51: " [...] remanet ipsa simplicissima entitas quae est essentia omnium, et non conspicimus ipsam talem entitatem nisi in doctissima ignorantia, quoniam cum omnia participantia entitatem ab animo removeo: nihil remanere videtur".

Cfr. DI, I, 16.

6 Como se sabe, Nicolás de Cusa ha denominado "Igualdad" a una de las Personas de la Trinidad: el Hijo o. Verbo de Dios (cfr. DI, I, 7, 19). En casi todas sus obras el autor ofrece denominaciones diversas para el Lógos: conceptus dei, praecisio, exemplar, veritas, rectitudo, mensura, perfectio, principium. El tema es siempre recurrente: en su consideración como forma complicans, esto es como la Foma única o Igualdad de todas las cosas, el Verbo resulta la clave para la comprensión - siempre ignorante - de la relación entre el Creador y la creatura, lo absoluto y lo contracto.

Cfr. DI, l, 17.

Cfr. Apologia doctae ignorantiae, n. 16.
} 
La explicación o difusión de esa forma única infinita en un mundo plural y finito implica, en primer lugar, la puesta en acto de solamente algunas de las infinitas posibilidades en una forma particular. Esta difusión de lo infinito recortada 0 actualizada es lo que el Cusano denomina "contracción" e implica, con relación al ser de las cosas en el Máximo absoluto, un empobrecimiento desde el punto de vista ontológico. Cada ente individual es un exponente único de un tipo particular de contracción, esto es de un peculiarísimo recorte en el despliegue de lo infinito en lo finito. Para el Cusano el término "contracto", pues, significa fundamentalmente "determinado"; ésta es la razón por la cual el universo es definido como máximo contracto: máximo en tanto uno; contracto, en cuanto ligado o vinculado por relaciones propias de determinación. Todo lo que proviene de la absoluta unidad es compuesto, por esto la unidad del universo no es absoluta sino una contracta unitas pues está en cada caso "contractada"por la composición, lo cual lo vuelve un infinito no en sentido absoluto o negativo sino en sentido privativo. ${ }^{10}$

El máximo contracto, pues, en cuanto principiado resulta explicatio dei, pero en cuanto segunda unidad o unidad de todos los contractos resulta una complicatio de la pluralidad. ${ }^{11}$ Vemos entonces que el esquema complicatio-explicatio no se aplica sólo a la relación entre lo increado y lo creado, sino que el Cusano lo utiliza "relativamente" en relación a la unidad-pluralidad del universo todo: sucesivas contracciones determinan las diversas y variadas "regiones del universo". ${ }^{12}$ Así se extiende la dialéctica complicatio-explicatio a otras unidades relativas en los diversos niveles de difusión o contracción de manera que permita esclarecer la singularidad. El mundo de la determinación se ofrece en una serie de unidades que se dan gradualiter y descendentes hasta alcanzar lo más contracto o determinado, el ente singular, en el cual se hallarán explicitadas o "explicadas" tales unidades sucesivas.

La relación principio-principiado se multiplica, pues, en términos relativos. En efecto, el universo todo o la unidad contracta de la pluralidad en cuanto principio de tal pluralidad debe estar presente en todas las cosas constituyendo, precisamente, su "ser contracto común" es decir no aquella que lo hace una con lo absoluto sino la que la hace una con el reino de la alteridad. Ahora bien, esta suerte de "quididad" no constituye, sin embargo, la esencia de los singulares. La alteridad no es nada ni en Dios ni fuera de Dios. ${ }^{13}$ Así pues, según el Cusano, la alteridad no puede ser esencia de ninguna $\operatorname{cosa}^{14}$ ya que más que una presencia señala una ausencia en cuanto que las determinaciones sucesivas implican una privación, un recorte de la infinita posibilidad en acto de la cual el mundo procede.

A propósito de esto dice Haubst, $R$ (Streifzüge in die cusanische Theologie, Münster, 1991, p. 199): "Die schöpferische Selbstentfaltung Gottes im Kosmos, oder: die alles Wirkliche umfassende Polarität von complicatio und explicatio ist sozusagen der weite Rahmen oder die grundperspektive, die im Hinblick auf die verschiedenen Regionen des Universums auf die je entsprechende Weise variiert".

13 Cfr. De vis.dei 14, 106. La alteridad no es en Dios en la medida que El sobrepasa toda oposicion y en él la diversidad es identidad. En cambio, la alteridad del mundo es aquella opuesta a la unidad.

14 De mente, VI: "alteritas ... de nullius rei essentia est". 
Hay, entonces, una sola forma para todas las cosas que puede ser llamada con propiedad "esencia" en el sentido de presencia: el máximo absoluto. La contracción sólo delimita en sentido privativo.

Las graduales y descendentes determinaciones en que la máxima unidad contracta o universo se hace presente en cada uno de los singulares son, a su tiempo, sucesivas unidades contractas desplegadas en cada singular. Por lo tanto, si bien lo único en acto es el singular, éste es el producto de una serie o cadena de contracciones: "Y así vemos cómo el universo por medio de tres grados se contrae en cualquier particular". ${ }^{15}$ En cada uno de estos grados son unidades contractas: en cuanto unidades "complican" la pluralidad unificándola, en cuanto contractas "explican" o explicitan, a su vez, la unidad de la cual proceden. Así, el universo que complica la pluralidad toda se explica en los géneros, los géneros en las especies. Sin embargo, lo único en acto son los individuos en los cuales "residen" contractamente todas estas unidades universales.

De esta manera, el compuesto fundante de todo individuo es esta presencia absoluta y su privación: el compuesto unidad-alteridad. De la composicion de la creatura pende la afirmación que Nicolás repite una y otra vez a lo largo de sus obras acerca de que todas las cosas son números es decir compuestos de unidad y alteridad. ${ }^{16}$ El mundo de la contracción, pues, es el ámbito donde es posible distinguir porque es el ámbito de lo vinculado, de lo susceptible de ser proporcionado, y el número es la herramienta fundamental que permite establecer las conveniencias y diferencias: "Acaso por esto Pitágoras juzgaba que todas las cosas se constituían y se entendían por la fuerza propia de los números". ${ }^{17}$

Llegados a este punto cabe preguntarse cómo responde Nicolás al problema de los universales. Indudablemente, lo expuesto da una respuesta al problema desde el punto de vista ontológico: la universalidad, concebida estrictamente desde la constitución ontológica de los entes, resulta una sucesión de unidades contractadas en el individuo: el universo todo, el género, la especie, las diferencias. Sin embargo, afirmamos en primer lugar que no se trataba de una presencia sino de una privación, y, en segundo lugar, que esta limitación o determinación es

${ }_{15}$ DI II, 6, 124.

${ }_{16}$ Por razones de extensión nuestro trabajo hace referencia sólo a este compuesto fundante. No obstante, cabe señalar que Nicolás de Cusa considera asimismo en cada ente singular la composición acto-potencia y la composición materia-forma; sin embargo resultan versiones de la dupla unidad-alteridad. La materia es posibilidad pura y nada en acto (DI II, 8 n 132: "Ouae (materia) nec aliquid nec nihil, neque una neque plures, neque hoc neque illud, neque quid neque quale, sed possibilitas ad omnia et nihil omnium actu". Sin embargo, no existe en cuanto posibilidad absoluta sino que siempre aparece contractada por el acto, pues considerada como posibilidad absoluta es Dios mismo. Asi, es ausencia de toda forma pero condición de existencia de toda forma: aún los entes incorpóreos tienen materia. En la naturaleza intelectual ésta es absorbida por la forma puesto que la unidad envuelve a la alteridad. Dios es en acto todo posible ser. En El la posibilidad no esta distinguida de la actualidad. La creatura, que no es todo lo que puede ser, siempre es compuesta. Sólo el hombre, en cuanto naturaleza intelectual, puede superar la composición pues es "imagen" de la unidad infinita.

Ya en el capítulo primero de DI (I, 1,3) se lee: "Proportio vero cum convenientiam in aliquo uno simul et alteritatem dicat, absque numero intelligi nequit. Numerus ergo omnia proportionabilia includit [...] Hinc forte omnia Pythagoras per numerorum vim constitui et intelligi iudicabat". 
única e irrepetible en cada singular. ¿Cómo se justifica, entonces, el universal predicable o lógico? En otros términos: si lo universal desde este punto de vista es, por definición, "lo que es predicado de muchos" ¿qué papel pueden desempeñar en cuanto fundamento ontológico de tal predicación estas determinaciones privativas que, además, son contractadas de tantas diversas maneras cuantos individuos haya?

Así como la doctrina cusana de la contracción da cuenta de la relación ontológica entre universalidad y singularidad; la doctrina cusana de la humana mens da cuenta de la posibilidad de la predicación universal.

En el marco del universo contracto aparece una presencia carente de toda contracción: la naturaleza intelectual o mens humana. Ella no es difusión o explicatio de la divina unidad sino simplemente su imagen; su alteridad en cuanto creada queda, pues, absorbida por la unidad participada de lo infinito que le es dada por el hecho de ser imago de la complicación eterna. ${ }^{18}$ Pero no es una imagen pasiva sino una viva imago ${ }^{19}$ que recorre la alteridad en busca de la armonía como una suerte de "número viviente" que opera distinciones para hallar las concordancias. ${ }^{20}$

La "regio humanitatis" gira en torno de esta potentia: la potencia que en ella procede de manera directa de la unidad y le permite "asimilarse" a toda multitud. ${ }^{21}$ La virtud o potencia humana es aquella que puede hacer aparecer aquella "esencia ausente de la alteridad", vale decir que es la única que puede establecer los vínculos de unidad en el marco de la determinación privativa. Esto es significativo no sólo de la activa capacidad del hombre sino que señala una exigencia de su propia existencia; en la potencia humana reposan Dios y el Universo: Dios, en tanto es imagen de su infinita unidad lo cual le confiere poseer una potencia semejante a la divina; pero también el Universo, puesto que es asimismo imagen de Dios en cuanto complicación absoluta de todo posible ser. Por esta última razón puede ser llamado "microcosmos"; por la primera "segundo dios" ${ }^{22}$ Esta potencia

18 Cfr. De mente, V. A propósito de este tema resulta imprescindible Das Menschenbild des Nikolaus von Kues und der Chrisliche Humanismus (MFCG 13, 1978), resultado de un Simposio llevado a cabo en Octubre del 77. En un trabajo anterior hemos abordado esto con mayor profundidad: D'Amico, Claudia, "Nicolás de Cusa, De mente: la profundización de la doctrina del hombreimagen" en Pat.et Med., XI (1991) p. 53-67. Cfr. De mente, V.

CFR. De mente, VII.

El clásico trabajo de Volkmann-Schluck (Nicolaus Cusanus. Die Philosophie im Übergang von Mittelalter zur Neuzeit, Frankfurt, 1957, Teil 3 ) se dedica muy especialmente a esta virtud "asimilativa" de la mens como esta posibilidad de asimilar la realidad a sí misma y hacerla una. En este sentido, hace notar la dependencia etimológica de "similis" respecto de "semel", es decir "lo que es del género de lo uno".

De coni. II, 14, 143: "Humanitatis igitur unitas cum humanaliter contracta existat: omnia secundum hanc contractionis naturam complicare videtur. Ambit enim virtus unitatis eius universa, atque ipsa intra sue regionis terminos adeo coercet: ut nihil omnium eius aufugiat potentiam, quoniam omnia sensu aut ratione aut intellectu coniectat attingi, atque has virtutes in sua unitate complicare dum conspicit se: et ad omnia humaniter progredi posse supponit. Homo enim deus est: sed non absolute: quoniam homo. Humanus est igitur deus. Homo etiam mundus est: sed non contracte omnia quoniam homo. Est igitur homo microcosmos aut humanus quidam mundus. Regio igitur ipsa humanitatis deum atque universum mundum humanali sua potentia ambit. Potest igitur homo esse humanus deus atque deus humaniter, potest esse humanus angelus, humana bestia, humanus leo aut ursus aut aliud quodcunque. Intra enim humanitatis potentiam omnia suo existunt modo". 
o vis propia es la que le permite establecer los lazos de la alteridad, esto es conferir una unidad a la alteridad que no es esencial como la divina, sino conjetural o "a la manera humana" (humaniter)..$^{23}$ Concebir, pues, es "hacer" las semejanzas de las cosas a través de las nociones de géneros, especies, diferencias. ${ }^{24}$

Todos los vocablos son impuestos por la fuerza de ratio que es, por definición, la facultad de la distinción. ${ }^{25}$ La razón da a cada cosa un nombre conveniente, sin embargo éste nunca será su nombre preciso. En efecto, si el nombre preciso de cada cosa es aquel que revela su esencia; siendo ésta una y la misma en todas las cosas, uno y el mismo será el nombre de todas. Más aún, bastaría conocer el nombre preciso de una para conocerlos todos; bastaria conocerlo, para conocer el máximo absoluto.

La mens humana ejerce un arte a la manera de la divina mens. Pero mientras que ésta es infinita, aquella es finita y, en cuanto opera "ratiocinando" se encuentra apresada en la distinción y determinación. Su tarea propia es fijar los límites de la alteridad nombrándola: al establecer un nombre la razón transforma lo que cae bajo los sentidos imponiendo la separación, la concordancia y la diferencia. Por este motivo, mientras que el arte de la complicación absoluta tiene el ejemplar único de todas las cosas, la imagen de esta complicación crea el ejemplar nocional de todas. ${ }^{26}$

Si esto es así hay un fundamento de la universalidad ante rem tal como lo demuestra la doctrina de la contracción pero hay una constitución post rem de la universalidad lógica, lo cual hace que el Cusano afirme sin vacilar que géneros y. especies, tal como caen bajo un vocablo, son entes de razón pues la razón los hace para sí a partir de la concordancia y la diferencia de lo sensible. ${ }^{27}$ En consecuencia, si géneros y especies son posteriores por naturaleza a lo sensible, entonces, destruídas las cosas sensibles, no pueden permanecer. ${ }^{23}$

Esta posición es considerada por el propio Nicolás como una suerte de sintesis concordante de las posiciones de "peripatéticos" y "académicos", en la medida en que considera los aspectos ante rem y post rem de la universalidad. ${ }^{29}$ Este camino que el Cusano cree de síntesis unifica la forma platónica y, a la vez, vitaliza el concepto del aristotelismo confiriéndole a la acción del espíritu humano mayor espontaneidad. ${ }^{30}$

23 No abordaremos aquí el sentido de la coniectura o scientia coniecturalis en Nicolás de Cusa. Sin embargo, los fundamentos de la misma han sido explicitados: la inaccesibilidad de la verdad en sí misma, el conocimiento racional limitado a la posibilidad de establecer proporciones, la matemática como el adecuado instrumento de la proporcionalidad.

Cfr. De mente, VIII

De mente, II: "Sunt enim vocabula motu rationis imposita".

$\mathrm{Ib}$.: "Si de vi vocabuli diligentius scrutandum est, arbitror vim illam, quae in nobis est, omnium rerum exemplaria notionaliter conplicantem..."

27 concordantia et differentia sensibilium".

Ib.: "Quare, cum sint posterius natura rebus sensibilius, quarum sunt similitudines, tunc sensibilibus destructis remanere nequeunt".

Ibidem.

Santinello lo resume magistralmente (Introduzione a Niccolò Cusano, Roma-Bari, 1987, p. 80): "La differenza fra la posizione platonica e quella aristotelica è resa, dunque, trasformando l'aristotelismo in un quasi-nominalismo. Però "Tutte queste differenze nella maniera di considerare le cose, e quante altre possano pensarsi, facilmente si resolvono in concordanza, quando la mente si elevi 
Hemos transitado, pues, el camino que discurre desde la caracterización cusana de la absoluta maximidad entendida como forma única universal al problema de la constitución de la trama nocional que vincula la alteridad mediante géneros y especies. Si bien no se considera la posición cusana en la llamada "querella de los universales", no podemos afirmar sin embargo que le fue totalmente ajena toda vez que creemos que puede ser llamado "problema de los universales" aquel que plantee la relación entre las palabras, los conceptos y las cosas. ${ }^{31}$

A la hora de definir la posición de nuestro filósofo creemos que conviene distinguir ciertos niveles en el abordaje de la cuestión. Por una parte, en el plano de la universalidad que podríamos llamar ontológica o, con más rigor, "teológicocausal" nada nos haría dudar en la ubicación de Nicolás de Cusa en el realismo más extremo. En efecto, hay una forma o principio único universal que oficia como esencia de lo real y que se halla a la vez "contractada" o contraída en la singularidad a través de unidades graduales y descendentes. Pero, por otra parte, el universal lógico o predicable es resultado de una acción directa de la razón que impone todo nombre $y$, en particular, el nombre universal mediante la vinculación de las semejanzas de los singulares. Estas concordancias no son, empero, una causa positiva sino privativa. El interés cusano reside en establecer el carácter conjetural de estas denominaciones universales pues las diferencias específicas indicadas por los nombres sólo señalan un producto del movimiento de la razón en su afán de tender lazos entre los singulares. El producto de este movimiento no es una diversidad de formas o esencias realmente existentes sino sólo una diversidad de ejemplares nocionales que permiten el conocimiento, siempre conjetural, de la multiplicidad y permiten, asimismo, la comunicación.

La quididad de cada cosa es el numerus de la mente divina. ${ }^{32}$ Este númeroesencia es sólo unidad; está desprovisto de composicion pero encierra toda la diversidad de la serie enumerable como identidad. Esta forma única es el nombre preciso de cada cosa sólo pensable cuando la mens, abandona la distinción y se eleva a lo infinito. Allí las diferencias coinciden, la pluralidad es una y sólo nos es posible una ignorancia docta.

all'infinito" (De mente, 2, §67). II Cusano, all'infinito, vede tutte le forme platoniche unificate nell'unica forma o Verbo divino; e le forme moltiplicarsi in re, ma rimanere inaccessibili e inconoscibili, come ogni essenza reale; i concetti aristotelici esser enti di ragione, coincidenti col contenuto nominale delle definizioni logiche, non certo con le forme".

"La querelle des universaux est une autre manière de dire les choses, les concepts et les mots" Alain de Libera, La querelle des universaux. De Platon à la fin du Moyen Âge, Paris, 1996, p. 14. En esta obra de reciente edición no se menciona la figura de Nicolás de Cusa.

De mente, VI: “... numerus divinae mentis" 